\title{
From Constituent Quark Structure to Hadron Structure Functions
}

\author{
Firooz Arash* \\ physics department, Tafresh University,Tafresh, Iran \\ E-mail.farashecic.aut.ac.ir
}

\section{Fatemeh Taghavi-Shahri}

Physics department, Iran University of Science and Technology, Tehran, Iran

\begin{abstract}
In the Framework of valon model Parton distributions in a valon is calculated for the polarized and unpolarized cases. They are originated purely from the dressing of a valence quark in QCD. Structure functions, $F_{2}^{p, \pi}$, are obtained, which agree rather well with the experimental data. A simple relation between $F_{2}^{p}$ and $F_{2}^{\pi}$ is inferred. For the polarized structure function, $g_{1}$ while the model gives good agreement with data, it requires a sizable angular momentum contribution to the spin of the valon, and hence to that of proton. This contribution is calculated.
\end{abstract}

International Europhysics Coference on High Energy Physics

July 21st - 27th 2005

Lisboa, Portugal

* Speaker. 
Parton distributions in proton have been studied extensively in a wide range $x$ and $Q^{2}$. They are calculated numerically by fitting over 1300 data points with over 30 input parameters. These PDF's are accurate but inconvenient to describe analytically. Recent data on pion [1], at very low $x$ serve as a further check on our understanding of hadron structure. Here we use valon model that can be very useful in the study of hadronic structure. A valon has its own cloud of partons that can be calculated in $\mathrm{pQCD}$. This structure is universal and independent of the hosting hadron. In [2] the structure of the valon is calculated to the Next-to-leading Order in QCD and tested against the $F_{2}^{p}\left(x, Q^{2}\right)$ in a wide range of kinematics: $x=\left[10^{-4}, 1\right]$ and $Q^{2}=[1,5000] \mathrm{GeV}^{2}$. Here we will use it to describe the pion structure functions, $F_{2}^{\pi}$ without any additional new parameter. This is important, because at first it is not clear at all that if one moves away from proton to pion, where the data are much restricted, the insight gained from the study of proton, will still be valid. At the end we will discuss the case of polarized structure function $g_{1}$.

(a) Unpolarized case The unpolarized structure of a valon in the NLO is given in Ref.[2]. It suffice here to give the parameterized form of the valonic PDF as follows: $\mathrm{z}$

$$
z q_{\text {sea }, \text { gluon }}^{\text {valo }}\left(z, Q^{2}\right)=\alpha z^{\beta}(1-z)^{\gamma}\left[1+\eta z+\xi z^{0.5}\right] . \quad z q_{\text {valence }}^{\text {valon }}\left(z, Q^{2}\right)=a z^{b}(1-z)^{c},
$$

The parameters $a, b, c, \alpha, \beta, \gamma, \eta$, and $\xi$ are functions of $Q^{2}$ (see appendix of Ref. [2].) Upon convolution of these valonic PDF's with the valon distribution, $G_{v a l o n}^{h}(y)$ in a hadron, we obtain the hadronic structure function:

$$
F_{2}^{p}\left(x, Q^{2}\right)=\sum_{\text {valon }} \int_{x}^{1} d y G_{\text {valon }}^{h}(y) F_{2}^{\text {valon }}\left(\left(\frac{x}{y}\right), Q^{2}\right)
$$

where $G_{v a l o n}^{h}(y)$ is the distribution of valon with momentum fraction $y$ in hadron h. These functions are given in Refs.[2] [3] [4] for proton, pion, and kaon. $F_{2}^{\text {valon }}$ is the structure function of a valon. In figures (9) of Ref.[2] the results for $F_{2}^{p}$ is presented for $1<Q^{2}<2000 \mathrm{GeV}^{2}$ and compared with the experimental values and other global fits, the agreements are excellent.

Pion structure function at low $x$ in the leading neutron experiment [1] is reported. There are also some data from Drell-yan [5] experiments at $x \geq 0.2$ for the valence quark distribution. We have used the data of Ref.[5] at $Q^{2}=25 \mathrm{GeV}^{2}$ to determine $G_{\text {valon }}^{\pi}(y)$. The results of the model is shown in Figure [1]. It turns out that the valon distribution in pion is very broad:

$$
G_{\frac{U}{\pi^{+}}(y)}=G_{\frac{\bar{D}}{\pi^{+}}(y)}=1.02(1-y)^{0.01} y^{0.01}
$$

This is expected; for it indicates that the valons in a pion are tightly bound, a reflection of the fact that the pion is much lighter than the mass of its valons. From Eqs.(1-3) $F_{2}^{\pi}$ is readily calculated without any free parameters. In Figures $(1,2)$ we present $F_{2}^{\pi}$ at $Q^{2}=7$ and $Q^{2}=15 \mathrm{GeV}^{2}$ at very low $x$. In the figure there are two sets of data points, corresponding to two different normalization of the data by ZEUS coll. They differ by a factor of two [1]. Our results favor the effective flux normalization. Same is true for SMRS , but GRS is more compatible with the additive quark model normalization. However, as can be seen from figure (1) GRS determination grossly disagrees with the valence quark distribution in pion. In [3] and [4] it is shown that the valon description holds true for all values of $Q^{2}$.

A simple relationship holds between $F_{2}^{p}$ and effective flux normalization of pion structure function, $F_{2}^{\pi(E F)}$, namely: $F_{2}^{\pi(E F)}\left(x, Q^{2}\right) \approx k F_{2}^{p}\left(x, Q^{2}\right)$, With $k \approx 0.37$. We have calculated both 

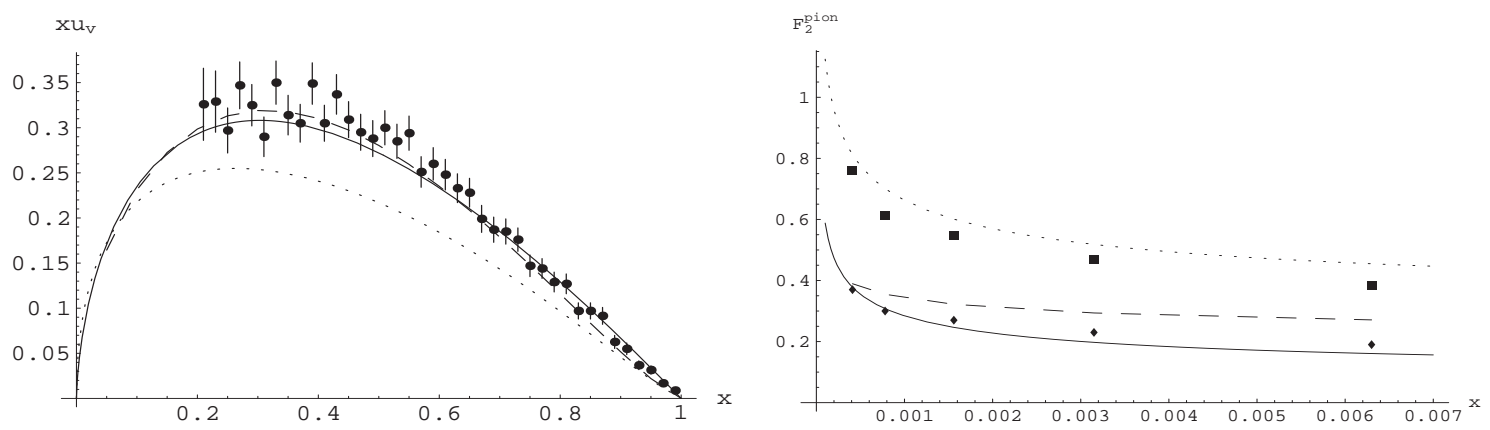

Figure 1: Right- Comparison of the pion valence distribution, $u_{\text {valence }}^{\pi^{-}}(x)$, using the valon model (solid line), SMRS (dashed line), and GRS (dotted line), with the data from Ref.[1] at $Q^{2}=25 \mathrm{GeV}^{2}$. Left- Pion structure function, $F_{2}^{\pi}(x)$, at $Q^{2}=7 \mathrm{GeV}^{2}$. The diamonds and the squares are pion flux and additive quark model normalization of the data [1], respectively. The solid line is the model calculation; the dashed line is from SMRS and the dotted line is from GRS determination.
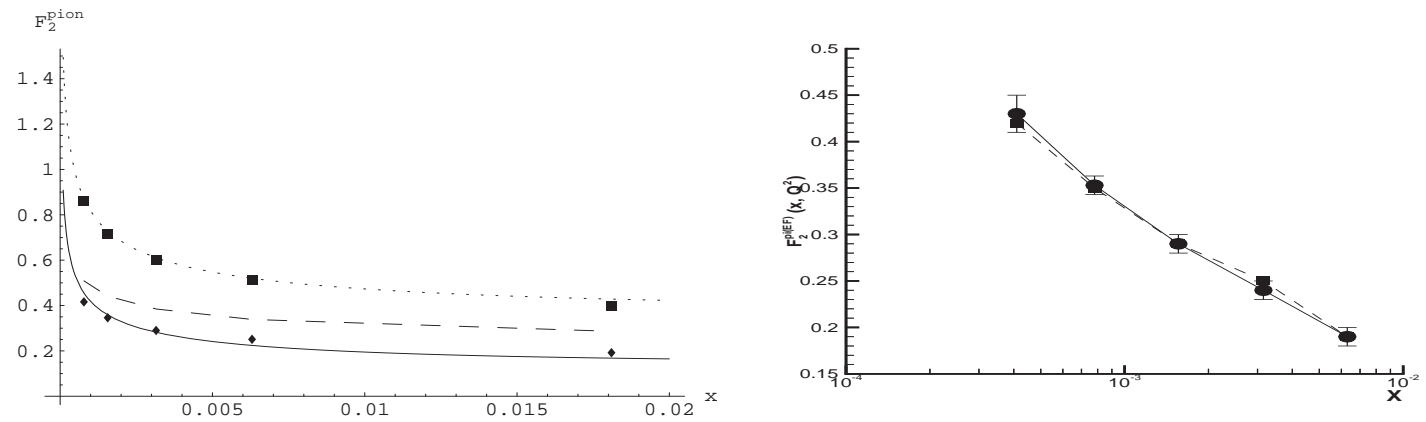

Figure 2: Right The same as the left figure in Figure-1 but at $Q^{2}=15 \mathrm{GeV}^{2}$. Left- The effective flux normalization of $F_{2}^{\pi}$ data as compared with the scaled $F_{2}^{p}$ at $Q^{2}=15 \mathrm{GeV}^{2}$. The data points are from Ref.[1]. The solid line is $0.371 F_{2}^{p}\left(x, Q^{2}\right)$ and the dashed line is $F_{2}^{\pi}$, both are calculated directly and separately from the model.

sides independently, using the valon model; a sample result at $Q^{2}=15 \mathrm{GeV}^{2}$ is given in Figure (3), but the relationships also hold true for other values of $Q^{2}$.

(b) Polarized Structure Functions The case of polarized structure function requires knowledge of polarized parton distribution in a polarized valon and the distribution of polarized valon in polarized hadron. We will restrict ourselves to nucleon. The first task is carried out similar to that of unpolarized case. for the second requirement, we define the function, $\delta F_{j}(y)$ via: $\delta G_{j}(y)=\delta F_{j}(y) G_{j}(y)$, where, $j$ refers to $\mathrm{U}$ or D valon, and $G_{J}(y)$ is the unpolarized valon distribution. In addition, we impose upon $\delta F_{j}(y)$, the constraint that $P_{U}=2 / 3$ and $P_{D}=-1 / 3$, corresponding to $S U(6)$ model. $P_{j}$ defined by $P_{j}=\int_{0}^{1} d y \delta F_{j}(y) G_{J}(y)$. For a nucleon, then we have:

$$
g_{1}^{N}\left(x, Q^{2}\right)=\int_{x}^{1} d y\left[2 \Delta G_{U} g_{1}^{U}\left(\frac{x}{y}, Q^{2}\right)+\Delta G_{D} g_{1}^{D}\left(\frac{x}{y}, Q^{2}\right)\right]
$$

This completes the formalism. In Figure (3) $x g_{1}^{p}$ are shown. It is evident that the model is able to reproduce the experimental data. Our findings for $\Gamma^{p(n)}=\int d x g_{1}^{p(n)}(x)$. are: $\Gamma^{p}=0.1212,0.1219,0.1225$, and 0.1236 for $Q^{2}=2,3,5,10 \mathrm{GeV}^{2}$, respectively, which are very close to the experimental data. The same is true for $\Gamma^{n}$. The model produces all available data on $g_{1}^{\text {p.n.d }}$, but the same data do not yield the nucleon spin. Therefore, it is natural to ask if the spin content of a valon do give the 

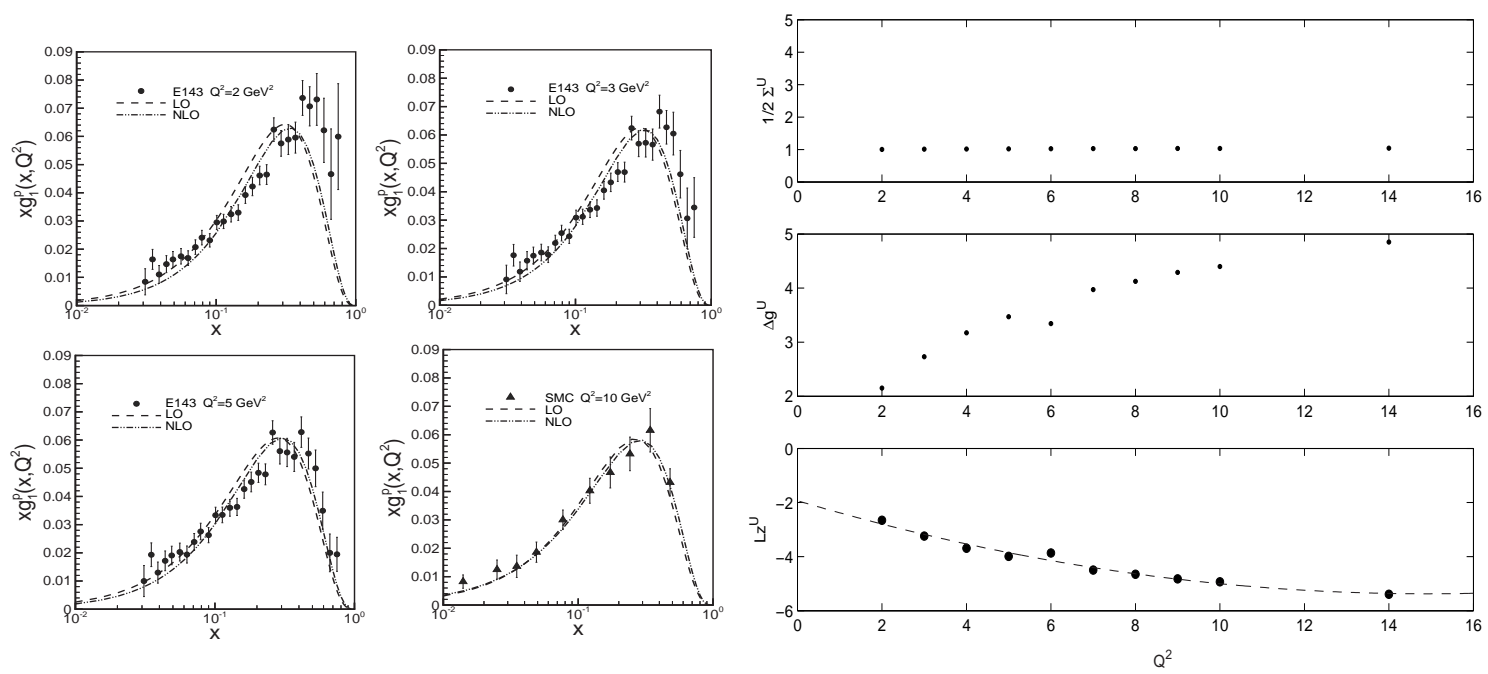

Figure 3: Right- $x g_{1}^{p}\left(x, Q^{2}\right)$ as calculated from the model and compared with the experimental data. Left- various component contributions to the spin of a valon as a function of $Q^{2}$

spin $\frac{1}{2}$ of the valon itself. The contribution of different components to the spin of, say a U-type valon, is given by the integral, $\Delta H_{\frac{i}{U}}\left(Q^{2}\right)=\int_{0}^{1} \delta q_{\frac{i}{U}}\left(z, Q^{2}\right) d z$; where $i$ is for valence, sea, and gluon inside a valon. The quark contribution $\frac{1}{2} \Delta \Sigma^{U}$ is constant for all values of $Q^{2}$, being equal to one. $\Delta H_{\frac{\text { gluon }}{U}}$, however, is fairly large and grows rapidly with $Q^{2}$, reaching to 4.5 at $Q^{2}=10 \mathrm{GeV}^{2}$. It is not,of course, unexpected according to QCD evolution equation. Thus, it is impossible to build a spin $\frac{1}{2}$ valon without an extra element; namely, the orbital angular momentum of sea partons in the valon, that enters the sum rule $S_{z}^{U}=\frac{1}{2}\left(S_{z}^{\text {valence }}+S_{z}^{\text {sea }}\right)^{U}+\left(S_{z}^{\text {gluon }}\right)^{U}+L_{z}^{U}=\frac{1}{2}$. These observations are shown in the left-side of Figure (3). There is a mixing operator which dresses any valence quark wave function with $\bar{q}-q$ pair to respect PCAC. The vacuum of these massive valons is a coherent superposition of cooper pairs of massless quark. This resembles the structure of a valon to that of superconductivity. In the anistropic superconductivity a particle of condensed fluid surrounded by a cloud of correlated particles must rotate around it with the axis of rotation $l$. The presence of anistropy leads to axial asymmetry of pairing correlations around the anistropy direction. The axis of asymmetry can be associated with the polarization vector of the valence quark located at the center of the cloud. The magnitude of this $l_{z}$ associated with the so-called back flow must be negative.This is precisely what we need, and obtained, to compensate the growth of gluon polarization.

\section{References}

[1] ZEUS Coll., S. Chrkanov,et al., Nucl. Phys. B 637(2002) 3.

[2] F. Arash, A. N. Khorramian, Phys. Rev. C 67 (2003) 045201.

[3] F.Arash, Phys. Lett. B 557 (2003) 38.

[4] F. Arash, Phys. Rev. D 679 (2004) 054024.

[5] J. S. Conway et al., Phys. Rev. D 39, 92 (1989). 